\title{
Analysis of the grapevine (Vitis vinifera L.) berry shape by using elliptic Fourier descriptors
}

\author{
P. BODOR ${ }^{1 *}$ (1), E. SOMOGYI ${ }^{1}$, L. BARANYAI ${ }^{2}$, J. LÁZÁR $^{3}$ and B. BÁLO ${ }^{1}$
}

${ }^{1}$ Department of Viticulture, Institute of Viticulture and Enology, Faculty of Horticultural Science, Szent István University, Budapest, Hungary

${ }^{2}$ Department of Measurements and Process Physics and Control, Institute of Biosystems Engineering and Process Control, Faculty of Food Science, Szent István University, Budapest, Hungary

${ }^{3}$ Research Institute for Viticulture and Oenology, National Agricultural Research and Innovation Centre, Kecskemét, Hungary

\section{CONFERENCE FULL PAPER}

Received: January 31, 2020 • Accepted: September 29, 2020

Published online: October 22, 2020

(C) 2020 The Author(s)

\begin{abstract}
Grapevine berry shape has important marketing value in the table grape commerce, hence variability evaluation of this characteristic is highly important. In this study berry shape of 5 table grape genotypes: "Fanny", "Lidi", "Hamburgi muskotály", "Moldova", and "Orsi" were compared. To evaluate the shape variability graphic reconstruction and elliptic Fourier analysis have been carried out. Shape outlines have been investigated and Principal Component Analysis (PCA) has been performed with the SHAPE software package. PCA of the contours showed that 6 out of the 77 principal components were effective to describe shape attributes. The first 6 PCsexplained $94.51 \%$ of the total variance. PC1 associated with the width and length of the berry. PC2 related to the shape of the top and bottom of the berries, while PC3 linked to the ratio of the top and the bottom width. ANOVA of the principal component scores revealed significant difference among the genotypes. Results suggest that morphology of the berry is a variable not only among but within the accessions. Our findings confirmed that elliptic Fourier descriptors (EFDs) would be a powerful tool for quantifying grapevine berry morphological diversity.
\end{abstract}

\section{KEYWORDS}

Vitis vinifera L., berry, morphology, shape, elliptic Fourier descriptor

${ }^{*}$ Corresponding author. E-mail: Bodor-Pesti.Peter@szie.hu 


\section{INTRODUCTION}

Table grape production is a rising sector of agriculture with increasing surface, which requires breeding of new cultivars (FAO-OIV, 2016). The most important targets of breeding programs are seedlessness, early ripening, abiotic stress resistance, and phenotypic appearance (Ferrara et al., 2017). Various berry shape types exist, and extreme ones have important marketing value. To define this phenotypic variability descriptor lists have already been developed. Pacottet (1905) distinguished 5 forms of the berries, while Bioletti (1938) classified cultivars into 15 classes. The most widely applied descriptor list given by the International Organisation of Vine and Wine (OIV) explains 10 types. It is difficult to precisely define the physical form of the berry, therefore reference cultivars are dedicated to each forms. For example, Riesling B has obloid berries, Chasselas B has globose, while Bicane has ovoid, etc. (OIV, 2009). In the case of organs with polygon shape attributes, e.g. leaves, landmarks serve geometric morphometric characterization (Bodor et al., 2018); contrary the berry shape is sphere or ellipsoid where the definition of homologous points is limited. Elliptic Fourier descriptors (EFDs) were developed for shape definition of closed contour (Kühl \& Giardina, 1982), where a chain-code is obtained from the outline of the object. Using the procedure leaf shape of grapevine accessions have already been described (Chitwood et al., 2014).

In this study the berry shape of 5 table grape accessions has been investigated with EFD in order to explore morphological variability and define the discriminative characters.

\section{MATERIALS AND METHODS}

Berry samples were provided by the Research Institute for Viticulture and Oenology of the National Agricultural Research and Innovation Centre (Kecskemét, Hungary). Grapevine accessions "Fanny", "Lidi", "Hamburgi muskotály", "Moldova”, and "Orsi" were investigated. Bunches were harvested in 2017 in full ripeness. Twenty not deformed berries of each cultivar were collected from the middle third of several bunches and stored in plastic bags until digitalization. Samples were removed from the clusters and pedicel was nipped at the surface of the berry. Berries were placed on a transilluminating LED light box and digitalized individually with a Sony A58 camera on ISO100. All samples were oriented in the same position with pedicel upward (Fig. 1). Pictures were then converted into bitmap (BMP) file format. Shape analysis was performed with the SHAPE software package according to the protocol published by Iwata and Ukai (2004). The software package contains 4 programs: ChainCoder converts the color image to black and white (binary), reduces the noise and provides the chain code of the object contour. Based on the chain code Chc2Nef program calculates the normalized EFDs. In our study orientation was standardized by the program according to the major axis of the first harmonic ellipse that corresponds to the first Fourier approximation. At the same time there is the possibility to standardize based on the longest radius and make manual definition of the starting point. PrinComp software performs the Principal Component Analysis (PCA) of the normalized EFDs based on the variance-covariance matrix of the coefficients. The number of analyzed harmonics were 20. In the last step PrinPrint software provides the contour reconstruction. Calculation of the elliptic Fourier coefficients according to the chain-code of the contours and analysis of the principal component scores of the provided data was carried out with the corresponding software of the SHAPE package. PCA scores, and 95\% ellipses were depicted. 


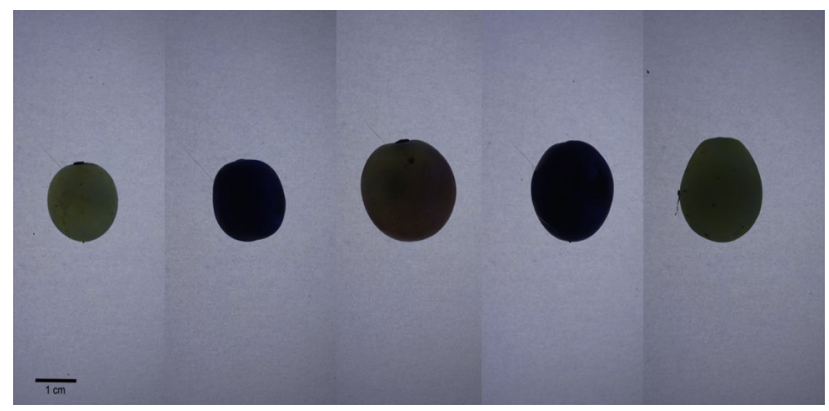

Fig. 1. Digitalized berry samples of "Fanny", "Hamburgi muskotály", "Lidi", "Moldova", and "Orsi" grapevine cultivars

Analysis of variance (ANOVA), post hoc test (Tukey) and cluster analysis based on the mean PC scores were carried out in the PAST 3.12. (Hammer et al., 2001). Digital reconstruction of the main shape types along the first 6 PCA was carried out in the SHAPE (Iwata \& Ukai, 2004).

\section{RESULTS}

Principal Component Analyis of the contours showed that 6 out of the 77 PCs were effective to describe shape attributes. The first 6 PC explained $94.51 \%$ of the total variance. PC1, PC2, and PC3 described 78.01, 6.10, and 4.67\%, respectively. PC1 associated with the width and length of the berry samples. Higher value of the PC1 are associated with the more globose (in terms of OIV, 2009), lower values with more narrow ellipsoid berries. PC2 and PC3 are related to the shape of the top and bottom of the berries, lower values are narrower at the bottom (ovoid) while higher values at the top (obovoid). (Fig. 2). Plotting the coordinates along PC1 and PC2 showed that "Orsi" has more narrow ellipsoid berries, while "Fanny" has more globose ones. Among the samples "Fanny" has wider berries at the bottom i.e. it is obovoid, while "Hamburgi muskotály" has them at the top, meaning it is more ovoid (Fig. 3).

ANOVA revealed significant difference among berry morphological characteristics of the accessions (Fig. 4). Three out of the 6 effective principal components were significantly different among the samples at $P<0.001$ level $(\mathrm{PC} 1: F(4,95)=42.23, P<0.0001$; PC2: $F(4,95)=5.55, P<$ 0.001; PC3: $F(4,95)=13.52, P<0.0001)$.

Morphological variability of the cultivars was evaluated based on the minimum and maximum PC values (data not shown). "Hamburgi muskotály" showed the largest morphological diversity along the PC1, while "Fanny" was the most uniform. Contrary to this, "Fanny" was the most variable along the PC2 and "Moldova" was the most uniform. In the case of PC3, "Lidi" showed the highest morphological diversity and "Fanny" was the most uniform. Along the PC4, "Moldova" was the most variable while "Orsi" had the lowest variability. Similarly to this, along PC5, "Moldova" was the most variable and "Orsi" was the most uniform. Samples obtained from "Lidi" proved to be the most divers along the PC6 while "Hamburgi muskotály" showed the lowest diversity.

Morphological similarity among the cultivars has been calculated by the mean values of the 6 PCs. Hierarchical clustering based on the Euclidean distance showed that "Lidi" and "Hamburgi 


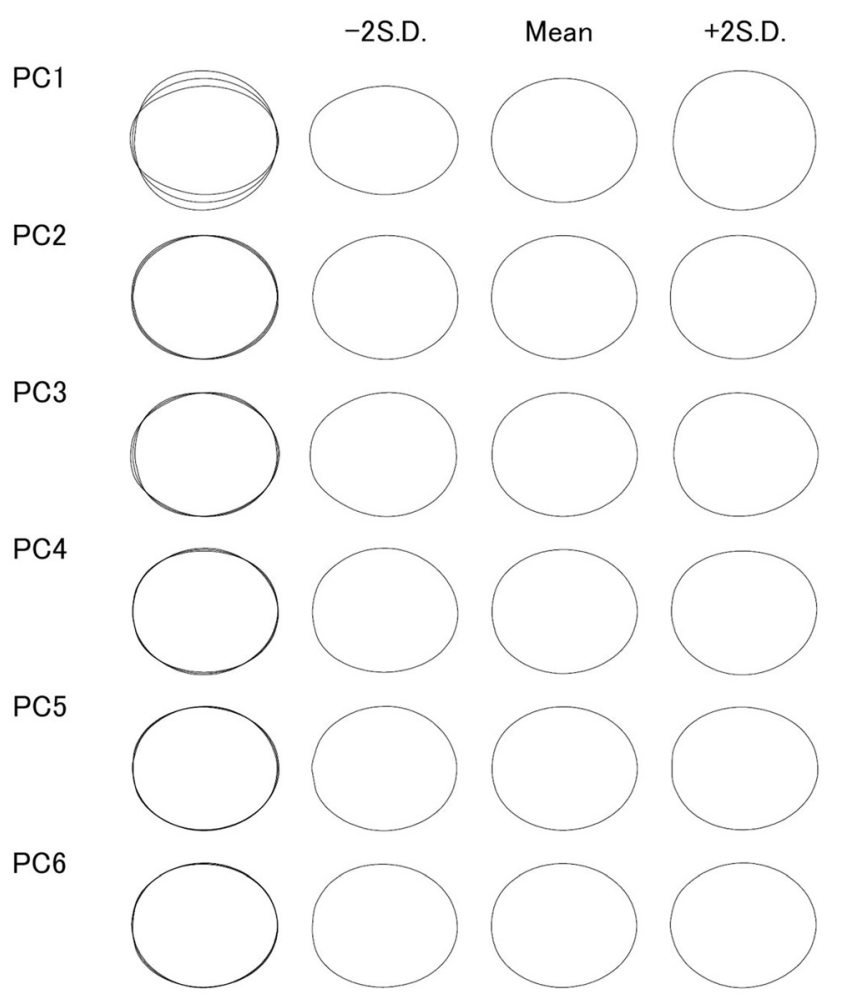

Fig. 2. Shape variation based on the 6 effective principal components representing the mean $-2 \mathrm{SD}$, mean and mean $+2 \mathrm{SD}$

muskotály" are the most similar in berry shape, while the most different are "Orsi" and "Moldova" (Fig. 5).

\section{DISCUSSION}

To describe and distinguish grapevine species and cultivars berry morphology has been in the focus of morphological characterization from the very beginnings. Worlidge (1691) has already mentioned the importance of berry size. Clemente (1807) classified the cultivars among others based on the berry shape. Reference shapes have been included in the ampelographic literatures to help description of the genotypes (Lauche \& Goethe, 1894).

During the 20th century with the help of photography new techniques were introduced in ampelography. Rodrigues (1953) for example used photograms to describe morphological diversity. In this study we recorded berry phenotype with digital photography.

Despite of the importance of berry shape its metric description (a.k.a. uvometry) is still come down to the measurement of the width and length or the ratio of these two, which is usually referred as the berry shape index. For example, El-Sayed (2013) reported data about the Crimson 


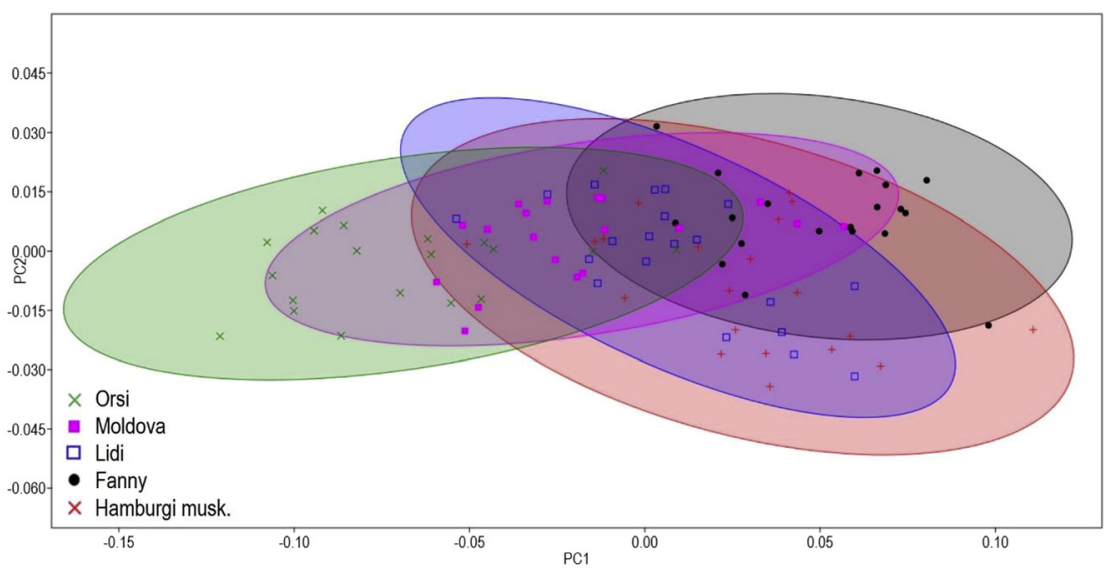

Fig. 3. PCA scores along PC1 and PC2
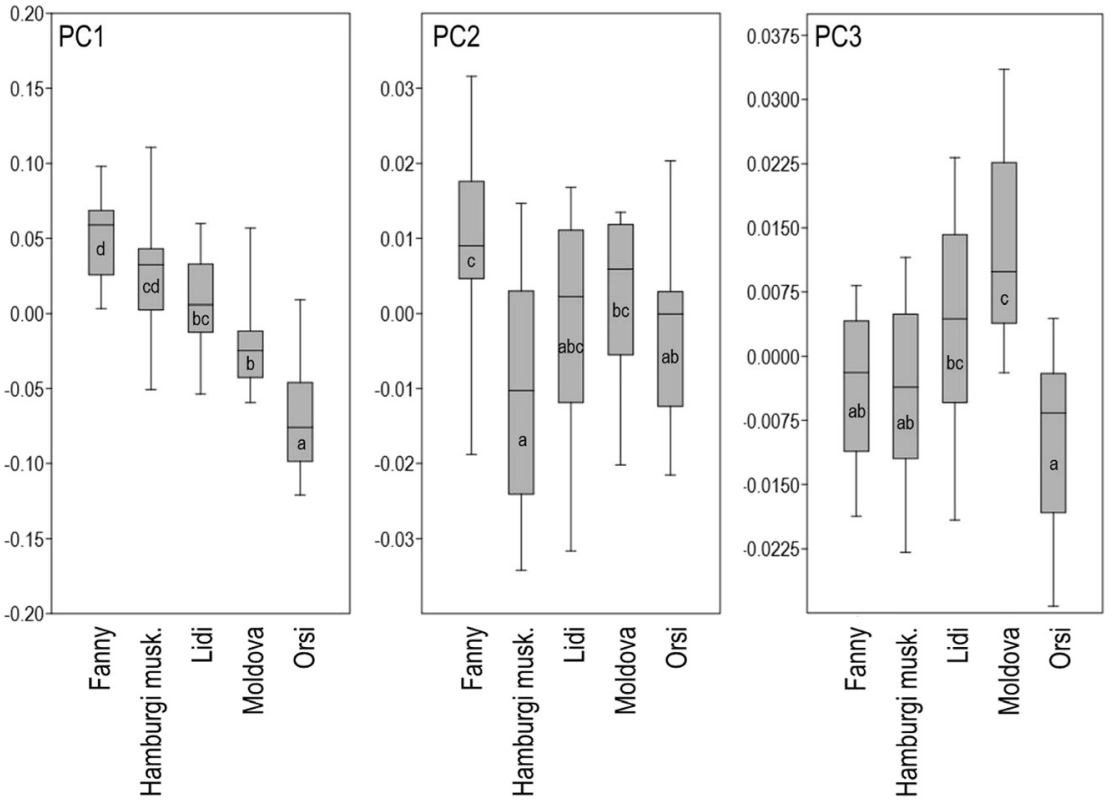

Fig. 4. Mean PC values of the investigated grapevine cultivars along PC1, PC2, and PC3 (letters indicating significant difference at $P<0.05$ )

Seedless grapevine berry shape based on this index. There are numerous scientific papers reporting about shape attributes of object with closed contour. One of the possible methods is the characterization based on EFDs. The process was introduced by Kühl and Giardina (1982) 


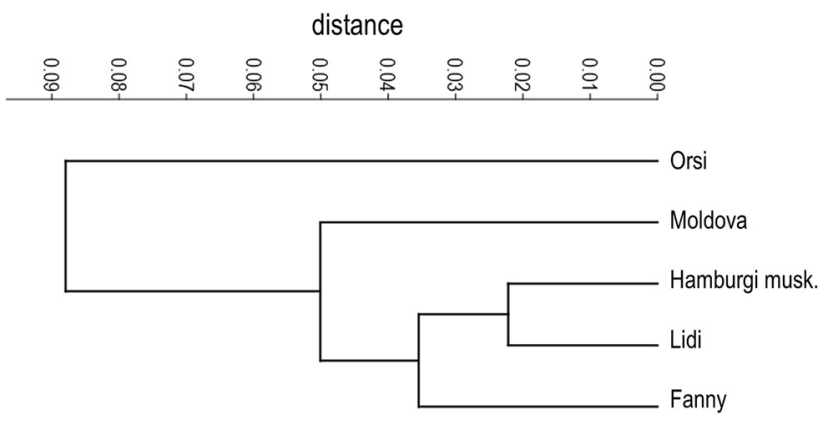

Fig. 5. Hierarchical clustering based on the mean scores of the first 6 PCs

and later applied in ampelography by Diaz et al. (1991) and Chitwood et al. (2014). In this study berry shape has been described by the method.

Results of this study showed that the berry shape is differing significantly among the genotypes. Also, the variability of the shape within the same genotype was not the same. This result is in accordance with the literature. Kozma (1968) mentioned that some of the cultivars have more uniform, while others have more variable berry shape. We found that "Hamburgi muskotály" has the highest morphological diversity while "Fanny" has the lowest. Our results show that the berry shape variability is an important phenomenon when evaluation of the consumer's preference is carried out. EFDs provide valuable information about the shape, at the same time size is also an important trait. Our next goal is to adopt further image analysis software already involved in the characterization of horticultural crops and describe large numbers of grapevine cultivars based on the size and shape attributes of the berry.

\section{CONCLUSIONS}

Grapevine berry shape is a variable morphological pattern whose description requires routine and reference cultivars. Our findings confirmed that EFD would be a powerful tool for quantifying berry morphological diversity.

\section{REFERENCES}

Bioletti, F.T. (1938). Outline of ampelography for the vinifera grapes in California. Hilgardia, 11(6): 227293, https://doi.org/10.3733/hilg.v11n06p227.

Bodor, P., Baranyai, L., Szekszárdi, A., Bisztray, Gy.D., and Bálo, B. (2018). Landmark-based morphometry reveal phyllometric diversity along the shoot axis of the grapevine (Vitis vinifera L.). Progress in Agricultural Engineering Sciences, 14(S1): 1-9, https://doi.org/10.1556/446.14.2018.S1.1.

Chitwood, D.H., Ranjan, A., Martinez, C.C., Headland, L.R., Thiem, T., Kumar, R., Covington, M.F., Hatcher, T., Naylor, D.T., Zimmerman, S., Downs, N., Raymundo, N., Buckler, E.S., Maloof, J.N., 
Aradhya, M., Prins, B., LI L., Myles, S., and Sinha, N.R. (2014). A modern ampelography: a genetic basis for leaf shape and venation patterning in grape. Plant Physiology, 164(1): 259-272. PMCID: PMC3875807.

Clemente, S.R. (1807). Ensayo sobre las variedades de la vid común que vegetan en Andalucía. Imprenta de Villalpando, Madrid. p. 324.

Diaz, G., Setzu, M., Diana, A., Loi, C., De Martins, B., Pala, M., and Boselli, M. (1991). Analyse de Fourier de la forme de la feuille de vigne. Première application ampelometrique sur un échatillon de 34 cépages implantés en Sardaigne. Journal International des Sciences de la Vigne et du Vin, 25: 37-49.

El-sayed, M.E.A. (2013). Improving fruit quality and marketing of "Crimson Seedless" grape using some preharvest treatments. Journal of Horticultural Science \& Ornamental Plants, 5(3): 218-226.

FAO-OIV (2016). Table and dried grapes. FAO-OIV Focus 2016. Non-alcoholic products of the vitivinicultural sector intended for human consumption. Published by the Food and Agriculture Organization of the United Nations and the International Organisation of Vine and Wine. p. 62. ISBN 978-92-5109708-3.

Ferrara, G., Gallotta, A., Pacucci, C., Matarrese, A.M.S., Mazzeo, A., Giancaspro, A., Gadaleta, A., Piazzola, F., and Colelli, G. (2017). The table grape 'Victoria' with a long shaped berry: a potential mutation with attractive characteristics for consumers. 15 June 2017. https://doi.org/10.1002/jsfa.8429. Published online in Wiley Online Library.

Hammer, O., Harper, D.A.T., and Ryan, P.D. (2001). PAST: Paleontological Statistics software package for education and data analysis. Paleontologia Electronica, 4(1): 9.

Iwata, H. and Ukai, Y. (2002). SHAPE: a computer program package for quantitative evaluation of biological shapes based on elliptic Fourier descriptors. Journal of Heredity, 93: 384-385.

Kozma, P. (1968). Csemegeszőlő. Mezőgazdasági Kiadó, Budapest, p. 451.

Kühl, F.P. and Giardina, C.R. (1982). Elliptic Fourier features of closed contour. Computer Graphics and Image Processing, 18: 236-258.

Lauche, W. and Goethe, R. (1894). Handbuch der Tafeltraubenkultur. Verlag von Paul Parey. Berlin, p. 235.

OIV (2009). OIV descriptor list for grape varieties and Vitis species, 2nd ed. Office International de la Vigne et du Vin, Paris, France.

Pacottet, P. (1905). Viticulture. Librairie Baillière, Paris, p. 483.

Rodrigues, M.A. (1953). Contribution à l'étude des rapports entre le volume et la forme des grains, le nombre et le poins des pépins, dans le Vitis vinifera L. VII. Congrés International de la Vigne et du Vin. Rome-Sienne, 13-20 Septembre 1953. 3: 107-127.

Worlidge, J. (1691). Vinetum Britannicum or a treatise of cider and other wines and drinks extracted from fruits growing in this Kingdom, London, p. 186.

Open Access. This is an open-access article distributed under the terms of the Creative Commons Attribution 4.0 International License (https://creativecommons.org/licenses/by/4.0/), which permits unrestricted use, distribution, and reproduction in any medium, provided the original author and source are credited, a link to the CC License is provided, and changes - if any - are indicated. (SID_1) 